\title{
Population Pharmacokinetics of S(-)-Carvedilol in Healthy Volunteers After Administration of the Immediate-Release (IR) and the New Controlled-Release (CR) Dosage Forms of the Racemate
}

Submitted: March 7, 2007; Accepted: May 10, 2007; Published: June 15, 2007

\author{
Ahmed A. Othman, ${ }^{1}$ David M. Tenero, ${ }^{2}$ Duane A. Boyle, ${ }^{2}$ Natalie D. Eddington, and Michael J. Fossler ${ }^{2}$ \\ ${ }^{1}$ Pharmacokinetics-Biopharmaceutics Laboratory, School of Pharmacy, University of Maryland, Baltimore, MD 21201 \\ ${ }^{2}$ Clinical Pharmacokinetics/Modeling and Simulation, GlaxoSmithKline, King of Prussia, PA 19406
}

\section{AbSTract}

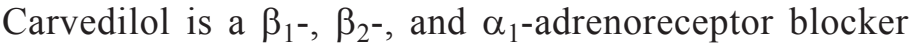
indicated for treatment of hypertension and mild-tosevere congestive heart failure. The objective of this study was to develop and evaluate a single population model that describes $S(-)$-carvedilol pharmacokinetics from both the immediate-release (IR) and the new controlledrelease dosage forms of the racemate. Carvedilol IR data (1270 measurements) were obtained from 2 open-label studies (50 mg/25 mg Q12 hours for 2 doses). Carvedilol CR data (2058 measurements) were obtained from an open-label, nonrandomized, dose-rising $(10,20,40$, and $80 \mathrm{mg}$ ), 4-period balanced crossover study. All data were simultaneously analyzed using NONMEM V. Leverage analysis and internal evaluations were conducted for the final model. A 2-compartment model with first-order absorption and elimination provided the best fit. The model included different absorption rates (KAs) for the $\mathrm{CR}$ and IR morning ( $\left.\mathrm{IR}_{\mathrm{AM}}\right)$ and evening ( $\left.\mathrm{IR}_{\mathrm{PM}}\right)$ doses; incorporating change-points at certain times. Estimates of KAs indicated that the absorption was slower at equivalent times and extended for $\mathrm{CR}$ relative to IR carvedilol. Oral clearance of $S(-)$-carvedilol was $149 \mathrm{~L} / \mathrm{h}$. The $\mathrm{IR}_{\mathrm{PM}}$ and the $\mathrm{CR}$ doses had bioavailability $\left(\mathrm{F}_{\text {rel }}\right)$ of 0.80 and 0.76 , respectively, relative to the $I_{\mathrm{AM}}$ dose. The inter-subject variability in KAs was lower for the CR dosage form than the original IR dosage form. Estimation of interoccasion variability on KAs and $F_{\text {rel }}$ for the CR dosage form improved the fit. The model performed well in simulation and leverage analysis indicated its robustness. The model will be a useful tool for future simulation studies.

KEYWORDS: Carvedilol, controlled-release, NONMEM, relative bioavailability, population analysis

Corresponding Author: Michael J. Fossler, GlaxoSmithKline, Clinical Pharmacokinetics Modeling and Simulation, 709 Swedeland Road, UW 2350, King of Prussia, PA 19406. Tel: (610) 270-4797; Fax: (610) 2705962; E-mail: Michael.J.Fossler@gsk.com

\section{INTRODUCTION}

Carvedilol is an $\alpha_{1}, \beta_{1}$, and $\beta_{2}$ adrenergic receptor antagonist. ${ }^{1-3}$ It is currently indicated for management of mild-to-moderate essential hypertension. In addition, carvedilol is proven, with chronic treatment, to reduce cardiovascular mortality and improve survival in patients with mild-to-severe heart failure and patients with systolic dysfunction after myocardial infarction. ${ }^{4-6}$ Carvedilol has 2 enantiomeric forms, $R(+)$ and $S(-)$. The $S(-)$ enantiomer is primarily responsible for the $\beta$-blocking effect of carvedilol, whereas both the $R(+)$ and $S(-)$ and enantiomers contribute to the $\alpha_{1-}$ blockade. ${ }^{7,8}$ Carvedilol undergoes significant stereoselective first-pass metabolism resulting in low absolute bioavailability upon oral administration (15.1 and $31.1 \%$ for the $S(-)$ and $R(+)$ enantiomers, respectively). ${ }^{9}$ The stereoselective metabolism of carvedilol is attributed mainly to CYP2D6 with higher metabolic stability of the $R(+)$ enantiomer when compared with the $S(-)$ enantiomer. ${ }^{9,10}$

The originally approved formulation of carvedilol by the U.S. Food and Drug Administration (FDA) is an immediaterelease (IR) dosage form of the racemate. The IR dosage form is administered twice daily to maintain the adrenergic antagonism over the 24-hour period. Recently, a controlled-release (CR) dosage form of carvedilol was developed to achieve drug exposure comparable to the IR dosage form but with only once-daily administration. Once-daily administration should improve patient compliance and ultimately maximize the beneficial clinical outcomes of carvedilol treatment.

The new CR dosage form is a capsule that contains anhydrous carvedilol phosphate microparticles formulated in the form of an IR component (12.5\%) and 2 micropumps, each coated with a $\mathrm{pH}$-sensitive polymer: Micropump IIa (37.5\% of the dose, designed to release its content at $\mathrm{pH} 5.5)$ and Micropump IIc (Flamel Technologies, SA, France) (50\% of the dose, designed to release its content at $\mathrm{pH}$ 6.4-6.8). ${ }^{11}$

The pharmacokinetics of the CR dosage form of carvedilol was evaluated and previously reported. ${ }^{11,12}$ The CR dosage form has shown a prolonged release profile as compared with the IR dosage form in healthy volunteers as well as in patients with hypertension and congestive heart failure. The bioavailability of the CR dosage form was lower than the IR dosage form at equivalent carvedilol free-base doses; necessitating 
$\sim 30 \%$ increase in the CR daily dose to achieve comparable exposure to the IR dosage form. The dose-exposure relationship for the CR dosage form was approximately linear over a range of 10 to $80 \mathrm{mg}$ of carvedilol phosphate ( 8.1 to $64.8 \mathrm{mg}$ of carvedilol free base). Carvedilol exposure was similar for the IR and CR dosage forms when administered with a standard meal (moderate calorie, low-to-moderate fat meal). In addition, the intersubject and intrasubject variability in the exposure parameters (eg, $\mathrm{AUC}$ and $\mathrm{C}_{\max }$ ) were comparable for the CR and IR dosage forms. Clinical evaluation of CR carvedilol in patients with essential hypertension indicated that once-daily administration of the CR dosage form provided effective blood pressure lowering over 24 hours with no indication of decreased efficacy or tolerability. ${ }^{13}$

The objective of the analysis presented in this article was to develop and evaluate a single population model that describes the pharmacokinetics of the IR and CR dosage forms of carvedilol in healthy volunteers. The model was developed to compare the absorption processes of both dosage forms as well as to estimate the intersubject variability in the pharmacokinetic parameters for both formulations.

\section{Materials ANd Methods}

\section{Design of the Studies Used in the Population Analysis}

The pharmacokinetic data analyzed were compiled from 3 studies in a total of 96 healthy volunteers. The IR data were obtained from 2 open-label studies where subjects were administered $50 \mathrm{mg}$ ( $25 \mathrm{mg}$ every 12 hours for 2 doses; dose strength based on the free base) of the commercially available immediate-release dosage form of carvedilol (COREG IR TILTAB [GlaxoSmithKline, Research Triangle Park, NC]) under fed conditions. The CR data were obtained from a nonrandomized, open-label, single dose, dose-rising, 4-period balanced crossover study. In the latter study, each subject received 4 doses $(10,20,40$, and $80 \mathrm{mg}$ of carvedilol phosphate; equivalent to $8.1,16.2,32.4$, and $64.8 \mathrm{mg}$ of carvedilol free base, respectively) of the controlledrelease dosage form under fed conditions with at least 1 week of wash-out between doses. Poor metabolizers of carvedilol were excluded during the enrollment phase by CYP2D6 genotyping. The 3 studies were conducted in accordance with all applicable regulatory guidelines and with the principles originating in the Declaration of Helsinki as amended in Hong Kong 1989. All subjects provided written informed consent before enrollment in the studies.

\section{Dosing Procedures}

In all studies, subjects fasted at least 8 hours before dosing. On the day of dosing, a moderate-calorie breakfast was given 30 minutes predose and the drug was administered orally with $240 \mathrm{~mL}$ of water within 5 minutes of completing break- fast. Lunch was served at $\sim 4$ to 5 hours post-dose while dinner was served $\sim 10.0-11.5$ hours postdose. Drinking water was allowed 2 hours after dosing. Soft drinks without caffeine or fruit juices (except grapefruit juice) were allowed starting 4 hours after dosing. Subjects were not allowed to drink grapefruit juice or eat grapefruit within 7 days before dosing and until collection of the final pharmacokinetic blood sample. Ingestion of caffeine- or xanthine-containing products as well as alcohol was not allowed for 24 hours before the start of the blood collections and during the sampling periods. Subjects were asked to maintain a sitting or supine/ semi-supine position for a minimum of 8 hours postdose.

\section{Blood Sampling}

Blood sampling for measurement of plasma carvedilol concentrations was conducted for each subject using an intravenous cannula inserted into an antecubital vein. For the IR dosage form, $\sim 3 \mathrm{~mL}$ of blood was collected predose and at 0.25 , $0.50,0.75,1,1.50,2,3,4,6,8,12,12.25,12.50,12.75,13$, $13.50,14,15,16,18,20,24,32$, and 48 hours after the morning administration. For the CR dosage form, samples were collected predose and at $0.25,0.50,0.75,1,1.50,2,3,4,5,6$, $7,8,10,12,18,24,32$, and 48 hours after administration.

\section{Analytical Methods}

Analysis of $S(-)$ carvedilol plasma concentrations was conducted using high-performance liquid chromatography/ tandem mass spectrometry (HPLC-MS/MS) as previously described. ${ }^{14}$ Briefly, $S(-)$ and $R(+)$ carvedilol were extracted from plasma by protein precipitation using acetonitrile containing racemic $\left[{ }^{2} \mathrm{H}_{5}\right]$-carvedilol as an internal standard. The extracts were then derivatized with the chiral derivatization reagent GITC (2,3,4,6-tetra-O-acetyl- $\beta$-D-glucopyranosyl isothiocyanate) and analyzed using HPLC-MS/MS with a turboionspray interface and selected reaction monitoring. Based on $150 \mu \mathrm{L}$ of plasma, the lower limit of quantification for $S(-)$ carvedilol was $0.2 \mathrm{ng} / \mathrm{mL}$ and linear responses in the analyte/internal standard peak area ratio were observed over a concentration range of 0.2 to $200 \mathrm{ng} / \mathrm{mL}$ with withinrun precision and bias and between-run precision of less than $15 \%$.

\section{Population Analysis}

\section{Model Building}

The IR and CR data were compiled and analyzed simultaneously using the nonlinear mixed effect modeling software NONMEM (version V, level 1.1; GloboMax LLC, Hanover, $\mathrm{MD})$. The concentrations of the $S(-)$ enantiomer of carvedilol were used for building the population model; consequently, all doses were adjusted during the analysis to reflect the 
administered amount of the free base of the $S(-)$ enantiomer. The actual sampling times rather than the protocol-defined times were used in the analysis. A 2-compartment structural model parameterized in terms of clearance (CL), volume of central compartment $(\mathrm{Vc})$, volume of peripheral compartment (Vp), intercompartmental clearance $(\mathrm{Q})$, and first-order absorption rate constant (KA) was used to fit the natural logtransformed concentrations of $S(-)$ carvedilol (ADVAN 4 TRANS 4 NONMEM subroutines). Addition of parameters to the base model was conducted in a stepwise manner. Noncompartmental analysis of the data indicated that the dose-normalized AUC for the CR dosage form and for the evening dose of the IR dosage form ( $\left.\operatorname{IR}_{\mathrm{PM}}\right)$ were lower relative to the IR morning dose $\left(\mathrm{IR}_{\mathrm{AM}}\right)$. Consequently, relative bioavailability factors $\left(\mathrm{F}_{\text {rel }}\right)$ were included in the model. $\mathrm{F}_{\text {rel }}$ was set to 1 for the $\mathrm{IR}_{\mathrm{AM}}$ dose (as the reference) and was estimated for the $\mathrm{IR}_{\mathrm{PM}}$ dose and for the CR dosage form. During the model-building process, different absorption rate constants $\left(\mathrm{KA}_{\mathrm{IR}}\right.$ and $\left.\mathrm{KA}_{\mathrm{CR}}\right)$ and different lag times (Tlag $\mathrm{IIR}_{\mathrm{R}}$ and Tlag $_{\mathrm{CR}}$ ) were assigned for the IR and CR dosage forms. Exploratory graphical analysis of the IR dosage form data indicated that the am and pm doses differ significantly in their absorption (Figure 1A). Consequently, different absorption rate constants were assigned for the $\mathrm{IR}_{\mathrm{AM}}\left(\mathrm{KA}_{\mathrm{IR}, \mathrm{AM}}\right)$ and the $\mathrm{IR}_{\mathrm{PM}}\left(\mathrm{KA}_{\mathrm{IR}, \mathrm{PM}}\right)$ doses. The graphical analysis also indicated that the absorption rate changes with time for the CR and IR dosage forms (Figure 1). As a result, the absorption rate constants were allowed to change at certain break points. Selection of the time and number of these break points was based on sensitivity analyses.

Intersubject variability in the pharmacokinetic parameters was estimated using an exponential error model as follows:

$$
P_{i}=T V P \exp \left(\eta_{i}\right)
$$

where $\eta_{\mathrm{i}}$ is the proportional difference between the hypothetical true parameter estimate of the $\mathrm{i}^{\text {th }} \operatorname{subject}\left(\mathrm{P}_{\mathrm{i}}\right)$ and the typical population parameter value (TVP) and is assumed to be normally distributed with a mean of 0 and a variance of $\omega^{2}$. Both common and different variances were evaluated to describe the intersubject variability in the absorption rate constants (KA) and in the relative bioavailability factors $\left(\mathrm{F}_{\text {rel }}\right)$.

To characterize the variability in $\mathrm{KA}_{\mathrm{CR}}$ and $\mathrm{F}_{\text {rel, } \mathrm{CR}}$ between the different $\mathrm{CR}$ study occasions (interoccasion variability), the following model was used as previously described ${ }^{15}$

$$
P_{i j}=T V P \exp \left(\eta_{i}+\kappa_{i j}\right)
$$

where $P_{i j}$ is the value of the $i^{\text {th }}$ subject parameter at the $j^{\text {th }}$ occasion and $\kappa_{i j}$ is assumed to be a normally distributed parameter with a mean of 0 and variance of $\pi^{2}$.

The residual error (which includes model misspecification and intrasubject variability, as well as errors in dosing, sampling times, and sample analysis) was evaluated using a logtransformed exponential error model as described by the following equation:

$$
\ln Y_{o b s}=\ln Y_{\text {pred }}+\varepsilon
$$

where $Y_{\text {obs }}$ is the observed plasma concentration, $Y_{\text {pred }}$ is the model-predicted plasma concentration, and $\varepsilon$ is a normally distributed parameter with a mean of 0 and variance of $\sigma^{2}$. In addition, the effect of incorporating intersubject variability in the residual error was evaluated using the previously described model ${ }^{16}$ :

$$
\ln Y_{o b s}=\ln Y_{\text {pred }}+\varepsilon \exp \left(\eta_{i}^{\mathrm{Re} s}\right)
$$

The first-order conditional estimation (FOCE) method with interaction between intersubject variability and residual variability was used throughout the model-building process.

Several criteria were used to evaluate the improvement in the model performance and to select the final model. The Likelihood Ratio Test was used for comparing rival hierarchical models where a decrease in NONMEM objective function
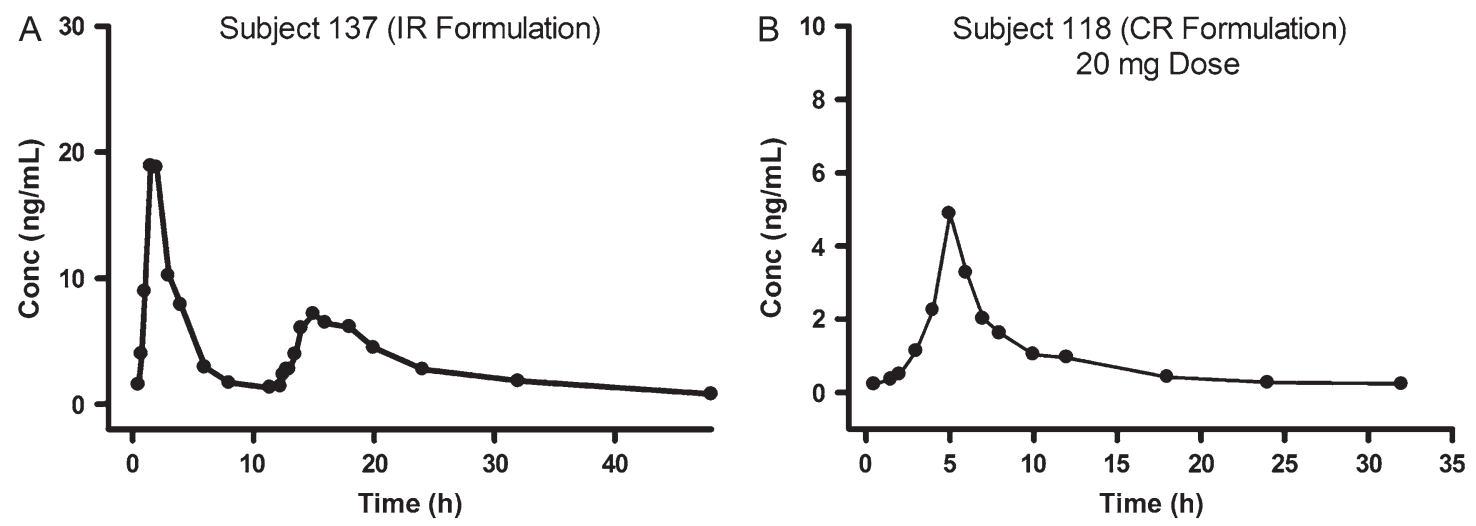

Figure 1. Representative plasma concentration (Conc) versus time profiles for $S(-)$ carvedilol in 2 of the healthy volunteers after administration of (A) $50 \mathrm{mg}$ of immediate release (IR) carvedilol (25 mg every 12 hours) or (B) $20 \mathrm{mg}$ of controlled release (CR) carvedilol. 
(-2 log likelihood) of 10.88 points was necessary to consider the improvement in model performance statistically significant at $\alpha=0.001$ and 1 degree of freedom. ${ }^{17}$ The Akaike information criterion (AIC) was used for comparing rival nonhierarchical models. ${ }^{18}$ The other selection criteria used included improved goodness of fit and residual plots, increased precision in parameter estimation, and reduced variance of intersubject and residual errors.

\section{Model Evaluation}

The final model was evaluated using (1) stepwise (one at a time) deletion of parameters and monitoring the impact on the model performance, (2) leverage analysis, and (3) internal evaluation using Monte Carlo simulation. The leverage analysis consisted of 10 runs. In each of these runs, $30 \%$ of the subjects were randomly removed without replacement from the whole data set using S-PLUS software (Insightful Corporation, Seattle, WA). The final model was then used to fit the remaining subset of the data, which included $70 \%$ of the subjects. This procedure was repeated 10 times with different subsets of subjects each created as described above. The mean and the coefficient of variation $(\% \mathrm{CV})$ of the parameter estimates from the 10 leverage analysis runs were then calculated and compared with the point estimates obtained using the complete data set. For the Monte Carlo simulations, Trial Simulator software version 2.1.2 (Pharsight Corporation, Mountain View, CA) was used to simulate the previously described IR and CR studies (500 replicates) using the final model. For each of the simulated replicates, the median $\mathrm{C}_{\max }, \mathrm{T}_{\max }$, $A \mathrm{AC}_{0 \text {-tlast }}$, and $\mathrm{C}_{\min }\left(\mathrm{C}_{12}\right.$ hour for $\mathrm{IR}_{\mathrm{AM}}$ dose and $\mathrm{C}_{24}$ hour for the $\mathrm{IR}_{\mathrm{PM}}$ and $\mathrm{CR}$ doses) were calculated at each dose for each dosage form. The 5 th and the 95 th percentiles (denoting the $90 \%$ prediction interval) of the medians of the parameters calculated for all the simulated replicates were then constructed. The same pharmacokinetic parameters were calculated for the actual data by noncompartmental analysis using WINNONLIN (Pharsight Corporation). The medians of the parameter estimates for the actual data were then compared with the constructed prediction intervals for the simulated data.

\section{RESULTS}

All 56 subjects enrolled in the IR studies (28 subjects/study) participated in both the am and pm dosing sessions. All 40

Table 1. Final Population Parameter Estimates for Analysis of the PK Data for the Controlled Release (CR) and Immediate Release (IR) Dosage Forms of Carvedilol

\begin{tabular}{|c|c|c|c|c|c|}
\hline \multicolumn{3}{|l|}{ Parameter } & $\begin{array}{c}\text { Point Estimate } \\
(\% \mathrm{RSE})\end{array}$ & $\begin{array}{l}\% \text { ISV* } \\
\text { (\%RSE) }\end{array}$ & $\begin{array}{l}\% \text { IOV }^{\dagger} \\
\text { (\%RSE) }\end{array}$ \\
\hline \multicolumn{3}{|l|}{$\mathrm{CL} / \mathrm{F}(\mathrm{L} / \mathrm{h})$} & $149(4.8)$ & ne & ne \\
\hline \multicolumn{3}{|l|}{$\mathrm{Vc} / \mathrm{F}(\mathrm{L})$} & $828(6.6)$ & $37.1(15.6)$ & ne \\
\hline \multicolumn{3}{|l|}{$\mathrm{Vp} / \mathrm{F}(\mathrm{L})$} & $1150(10.3)$ & ne & ne \\
\hline \multicolumn{3}{|l|}{$\mathrm{Q} / \mathrm{F}(\mathrm{L} / \mathrm{h})$} & $94.7(5.3)$ & ne & ne \\
\hline \multirow[t]{7}{*}{$\mathrm{KA}\left(\mathrm{hr}^{-1}\right)$} & $\mathrm{CR}$ & $0-2 \mathrm{~h}$ & $0.08(16.0)$ & & \\
\hline & & $2-4 \mathrm{~h}$ & $0.27(16.1)$ & $94.6(33.0)$ & $113.6(24.1)$ \\
\hline & & $>4 \mathrm{~h}$ & $3.5(17.7)$ & & \\
\hline & $\mathrm{IR}_{\mathrm{AM}}$ & $0-1 \mathrm{~h}$ & $0.92(21.5)$ & $140.4(20.4)$ & ne \\
\hline & & $>1 \mathrm{~h}$ & $8.79(42.1)$ & & \\
\hline & $\mathrm{IR}_{\mathrm{PM}}$ & $0-1 \mathrm{~h}$ & $0.42(26.7)$ & $193.1(17.8)$ & ne \\
\hline & & $>1 \mathrm{~h}$ & $3.0(31.9)$ & & \\
\hline \multirow[t]{4}{*}{$\mathrm{F}_{\text {rel }}$} & $\mathrm{CR}$ & & $0.76(7.4)$ & $33.8(14.1)$ & $14.1(67.3)$ \\
\hline & $\mathrm{IR}_{\mathrm{AM}}$ & & 1 (Fixed) & & \\
\hline & $\mathrm{IR}_{\mathrm{PM}}$ & & $0.80(3.2)$ & & \\
\hline & $\mathrm{CR}$ & & 0.23 (Fixed) & ne & ne \\
\hline Tlag (h) & IR & & $0.2(5.3)$ & & \\
\hline \multicolumn{3}{|c|}{$\sigma^{2}$ (Residual Error) } & $0.10(5.8)$ & $21.95(27.4)$ & ne \\
\hline
\end{tabular}

PK indicates pharmacokinetic; \%RSE, percent relative standard error; CL, clearance; Vc, volume of central compartment; Vp, volume of peripheral compartment; Q, intercompartmental clearance; KA, first-order absorption rate constant; $F_{\text {rel }}$, relative bioavailability factors; Tlag, lag time; ne, not estimable.

${ }^{*} \% \mathrm{ISV}\left(\%\right.$ intersubject variability) for a parameter $\mathrm{P}$ was calculated as $\omega_{\mathrm{P}} * 100$.

$\dagger \% \mathrm{IOV}$ (\% interoccasion variability) for a parameter $\mathrm{P}$ was calculated as $\pi_{\mathrm{P}} * 100$. 
subjects enrolled in the CR study participated in the 4 sessions for the rising doses except for 2 subjects where one dropped out after the 10-mg dose and the other dropped out after the 40-mg dose. A total of 3328 plasma $S(-)$ carvedilol concentrations were available for the population analysis with the IR studies contributing 1270 concentrations and the CR study contributing 2058 concentrations. The lowest and highest concentrations were 0.2 and $40.4 \mathrm{ng} / \mathrm{mL}$ for the IR dosage form and 0.2 and $42.4 \mathrm{ng} / \mathrm{mL}$ for the CR dosage form, respectively. The minimum, maximum, and average numbers of samples per subject were 18.0, 24.0, and 22.6 for the IR dosage form and 10.0, 63.0, and 51.4 for the CR dosage form, respectively.

\section{Model Building}

Based on preliminary analysis of the data and previous modeling experience with carvedilol, a 2-compartment structural model was used as the base model. However, the basic model was too simple to describe the combined IR and CR data (NONMEM minimization terminated) and incorporation of bioavailability factors relative to the IR am dose was necessary to achieve successful NONMEM con- vergence. Assigning dosage form-dependent absorption rate constants $\left(\mathrm{KA}_{\mathrm{CR}}\right.$ and $\left.\mathrm{KA}_{\mathrm{IR}}\right)$ resulted in a significant drop in NONMEM objective function $(\triangle \mathrm{OF}=-108)$. Further, partitioning of $\mathrm{KA}_{\mathrm{IR}}$ into am and pm components as well as incorporation of different lag times for the IR and $\mathrm{CR}$ dosage forms significantly improved the fit to the data $(\Delta \mathrm{OF}=-230)$. Estimation of the lag time for the $\mathrm{CR}$ dosage form was not possible as it resulted in rounding errors during the minimization process (NONMEM error code 134); therefore, Tlag $_{\mathrm{CR}}$ was fixed based on a sensitivity analysis. During that analysis, the impact of fixing Tlag $_{\mathrm{CR}}$ to different time values spanning the interval between administration of the CR dosage form and the time at which significant carvedilol levels were detected in plasma was examined (results not shown). Based on the sensitivity analysis, $\operatorname{Tlag}_{\mathrm{CR}}$ was fixed to 0.23 hour since this value resulted in the lowest NONMEM objective function. Further partitioning of Tlag for the IR dosage form into am and pm components did not improve the fit significantly and therefore was not applied. As expected from the trends in the data, allowing the absorption rate constant to change at certain time points dramatically improved the fit to the data. Consequently, a series of sensitivity analyses were conducted to choose the time and frequency of change of KAs and the drop in NONMEM
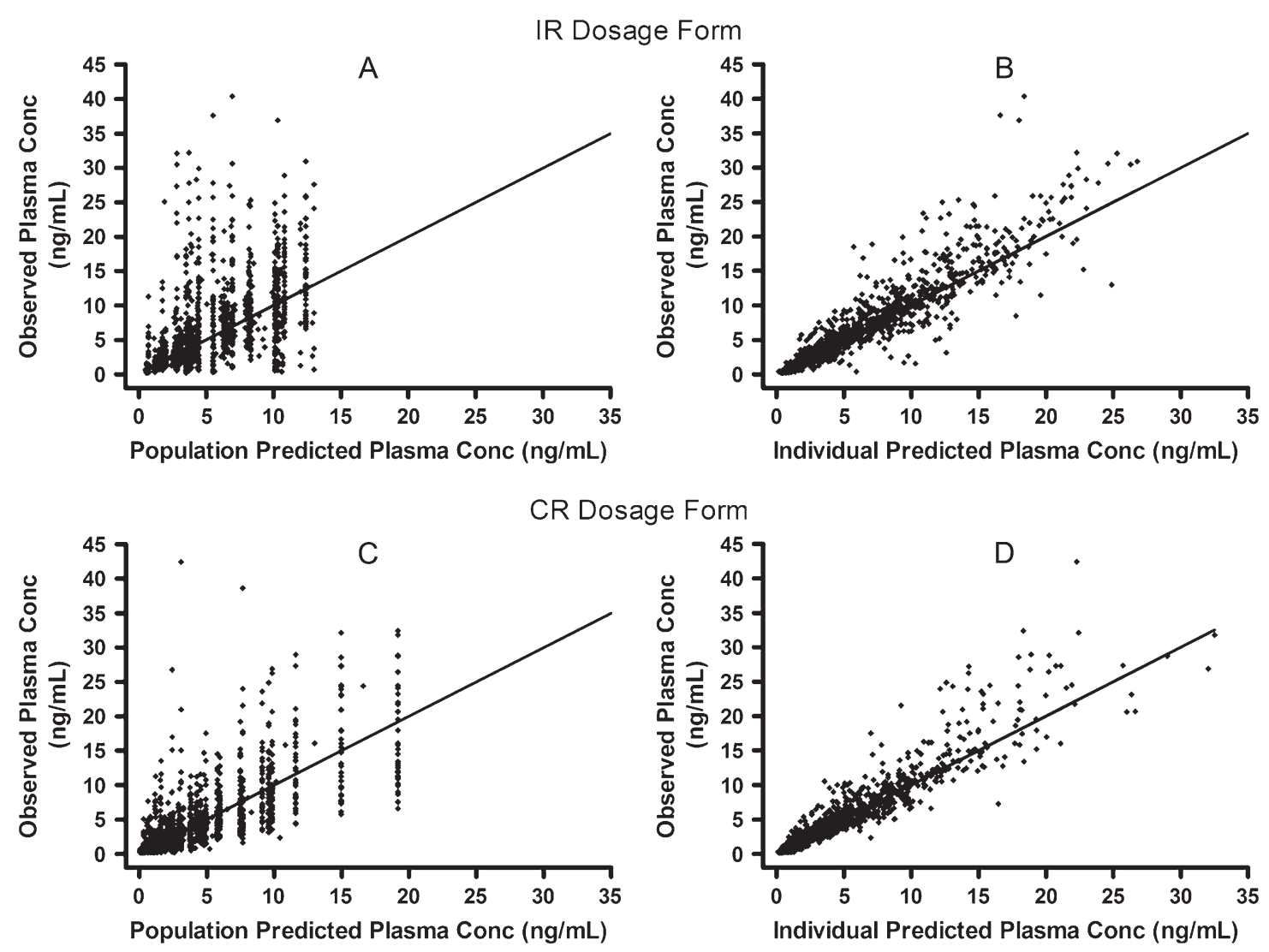

Figure 2. Scatter plots of observed versus population (A and C) or individual (B and D) predicted $S(-)$ carvedilol plasma concentrations stratified by dosage form. The plots are based on the fit achieved with the final model and the solid lines are the lines of identity. IR indicates immediate release; CR, controlled release; Conc, concentration. 
objective function was used as the judging criterion (results not shown). The results of the sensitivity analyses indicated that 2 break points for $\mathrm{KA}_{\mathrm{CR}}$ at 2 and 4 hours postdose and 1 break point for each of $\mathrm{KA}_{\mathrm{IR}, \mathrm{AM}}$ and $\mathrm{KA}_{\mathrm{IR}, \mathrm{PM}}$ at 1 hour postdose were optimal to describe the trends in the data $(\Delta \mathrm{OF}=-994)$. During the model development, incorporation of intersubject variability on $\mathrm{Cl} / \mathrm{F}, \mathrm{Vp} / \mathrm{F}$, and $\mathrm{Q} / \mathrm{F}$ did not significantly improve the fit $(P>.05)$, and as a result they were omitted from the model. Describing intersubject variability in $\mathrm{KA}_{\mathrm{CR}}, \mathrm{KA}_{\mathrm{IR}, \mathrm{AM}}$, and $\mathrm{KA}_{\mathrm{IR}, \mathrm{PM}}$ using different variances resulted in significant improvement in model performance $(\mathrm{OF}=-1328)$ when compared with either lumping the three $\mathrm{KAs}(\mathrm{OF}=-659)$ or lumping $\mathrm{KA}_{\mathrm{IR}, \mathrm{AM}}$ and $\mathrm{KA}_{\mathrm{IR}, \mathrm{PM}}$ with a common variance (minimization terminated with $\mathrm{OF}=-681)$. On the other hand, a common variance for describing the intersubject variability in $\mathrm{F}_{\text {rel } \mathrm{CR}}, \mathrm{F}_{\text {rel IR,AM }}$

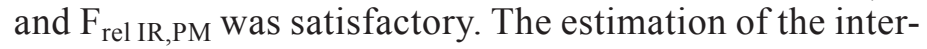
subject variability in Tlag was not possible. A log-transformed exponential residual error model that incorporated intersubject variability on $\varepsilon$ was found to describe the error in the data better than the model lacking the intersubject variability term $(\Delta \mathrm{OF}=-290)$. Finally, modeling the inter-occasion variability in $\mathrm{KA}_{\mathrm{CR}}$ and $\mathrm{F}_{\text {rel } \mathrm{CR}}$ significantly reduced the objective function as well as the magnitude of the residual random error $(\triangle \mathrm{OF}=-872)$.
The parameter estimates from the final model along with their associated \% relative standard errors (\%RSE) are shown in Table 1 . The model had 25 parameters that were all described with adequate precision. Except for the KAs, the fixed effect parameters were estimated with \%RSE of $\leq 10.3 \%$. All the different absorption rate constants (KAs) were estimated with \%RSE ranging from $16.0 \%$ to $42.1 \%$. The random effect parameters (intersubject, interoccasion, and residual variability) were estimated with \%RSE ranging from $5.8 \%$ to $67.3 \%$. The dosage form stratified scatter plots of the observed versus population predicted concentrations (Figures 2A and 2C), observed versus individual predicted concentrations (Figures $2 \mathrm{~B}$ and 2D), and observed and individual predicted concentrations versus time (Figure 3 ) indicated that the model adequately described $S(-)$ carvedilol plasma concentrations over the entire range for both the IR and CR dosage forms. In addition, the dose-stratified scatter plots of the observed versus individual predicted concentrations for the $\mathrm{CR}$ dosage form indicated that the model performed reasonably well for all 4 CR doses (Figure 4). The scatter plots of the weighted residuals versus population predicted concentrations or square root of time did not show any pattern indicating the lack of any systematic bias in the fit to the data for the IR (Figures 5A and 5B) and $\mathrm{CR}$ (Figures 5C and 5D) dosage forms. In addition,
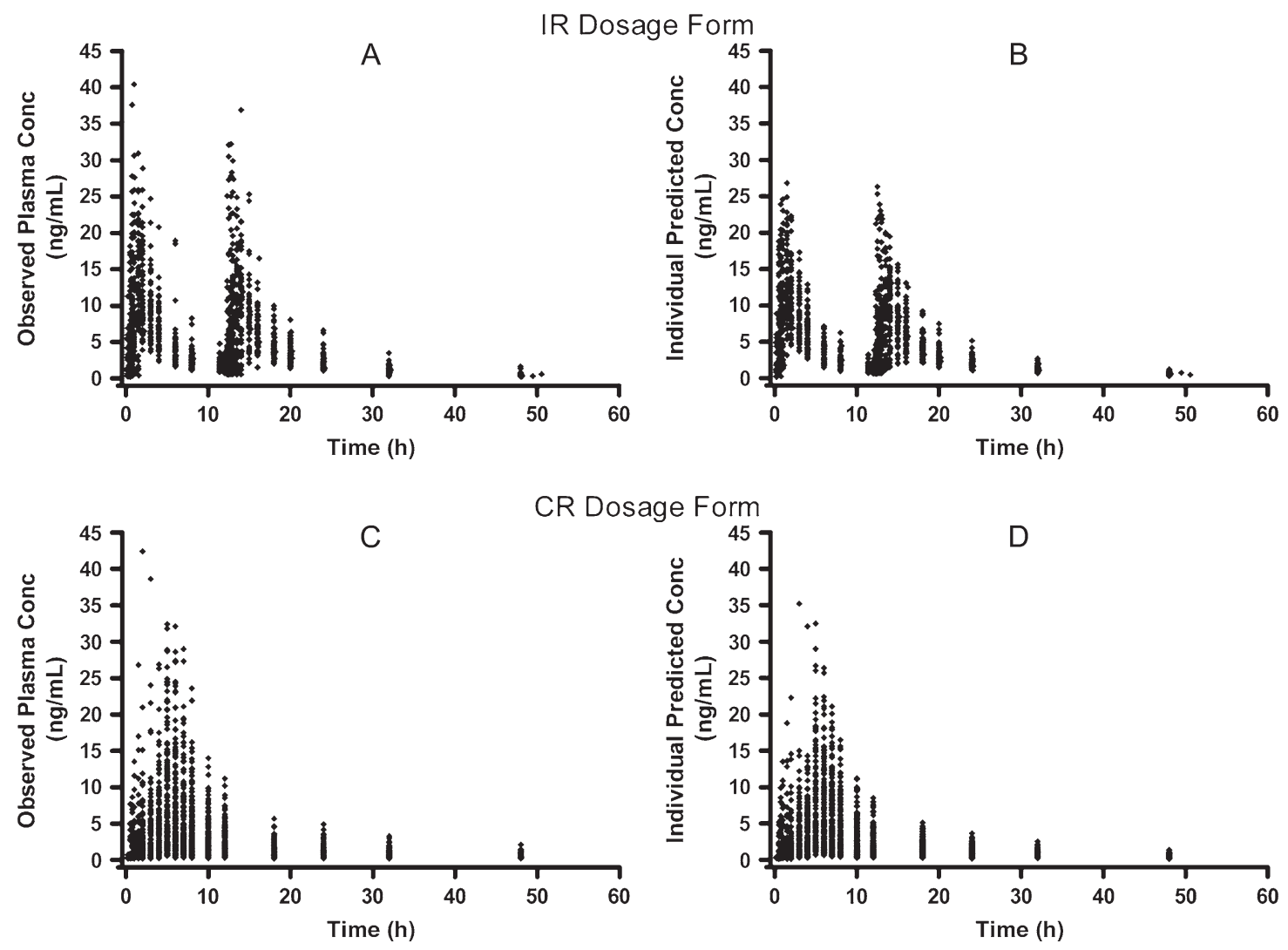

Figure 3. Observed (A and C) and individual predicted (B and D) $S(-)$ carvedilol plasma concentrations versus time profiles stratified by dosage form. IR indicates immediate release; CR, controlled release; Conc, concentration. 
The AAPS Journal 2007; 9 (2) Article 23 (http://www.aapsj.org).
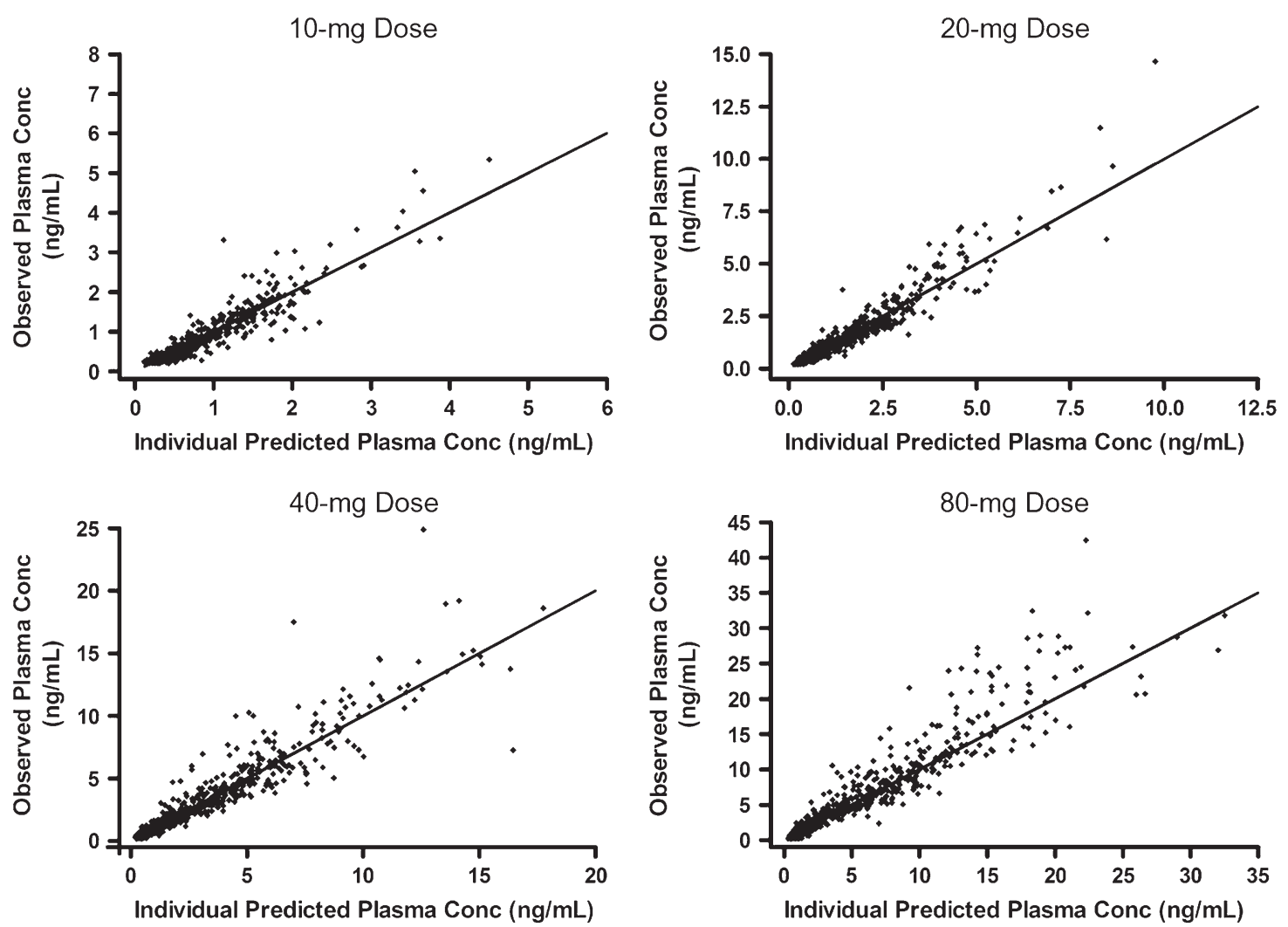

Figure 4. Observed versus individual predicted $S(-)$ carvedilol plasma concentrations (Conc) for the controlled-release dosage from stratified by dose. The solid lines are the lines of identity.

$97.5 \%$ of the weighted residuals were within $\pm 3 \mathrm{SD}$ of the mean (Figure 5). ETA matrix plots did not show any trends of correlation between the estimated intersubject variability parameters (not shown).

\section{Model Evaluation}

The results of the stepwise deletion of parameters from the final model are presented in Table 2. Deletion of each parameter resulted in an increase in the objective function that exceeded the minimum value defined by the proper test (Likelihood Ratio Test or AIC criterion) for statistical significance. Therefore, all the parameters included in the final model were necessary to fit the trends in the data. The results of the leverage analysis are presented in Table 3. The mean of the parameter estimates from the 10 runs were comparable to the final model estimates. In addition, the variability among the runs (as demonstrated by the $\% \mathrm{CV}$ ) was fairly low for most of the parameters, which indicates that the final model was robust and that the parameter estimates were not significantly driven by any particular portion of the data. The results of the internal evaluation of the predictive ability of the model using Monte Carlo simulations are presented in Table 4. All medians of $\mathrm{AUC}_{0 \text {-tlast }}, \mathrm{C}_{\max }, \mathrm{T}_{\max }$, and $\mathrm{C}_{\min }$ calculated for the actual observations fell in the $90 \%$ prediction interval limits for the medians of the 500 replicates of simulated data except for the $\mathrm{C}_{\max }$ of the IR am dose where the difference was less than $0.6 \mathrm{ng} / \mathrm{mL}$ (Table 4).

\section{DISCUSSION}

A new controlled-release dosage form of carvedilol has been recently developed and approved by the FDA for management of mild-to-moderate essential hypertension and for treatment of patients with mild-to-severe congestive heart failure and patients with post-myocardial infarction left ventricular dysfunction with or without symptomatic heart failure. In this article, the development and evaluation of a single population model for the pharmacokinetics of $S(-)$ carvedilol in both the original IR dosage form and the recently developed $\mathrm{CR}$ dosage form were described. The population analysis focused on the concentrations of $S(-)$ carvedilol because it is the enantiomer responsible for the beta-blocking activity of carvedilol.

During the analysis, a trend of change in the absorption pattern of carvedilol with time was observed and this trend was more prominent with the CR dosage form. This change was consistent with the fact that the CR dosage form is composed of 3 components ( 1 IR portion and 2 CR micropumps) 

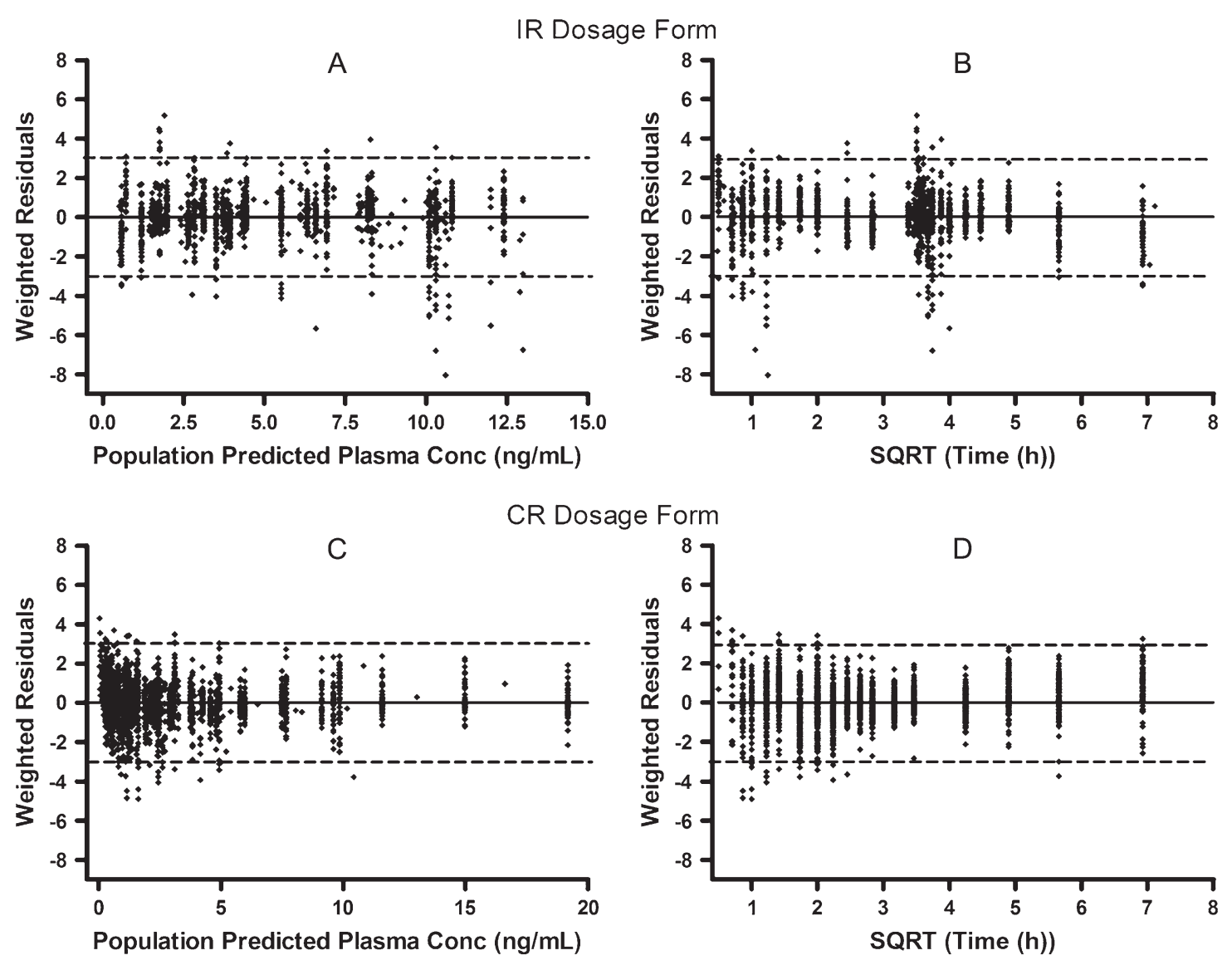

Figure 5. Scatter plots of the weighted residuals versus population predicted $S(-)$ carvedilol plasma concentrations (A and C) or square root of time (B and D). The plots are stratified by dosage form and the dashed lines represent $\pm 3 \mathrm{SD}$. IR indicates immediate release; $\mathrm{CR}$, controlled release.

designed to release their contents in a $\mathrm{pH}$-dependent sequential manner. Consequently, to be able to model the absorption process adequately, the absorption rate constants were allowed to change at certain break points and this divided the absorption process into different stages. In the final model, the absorption phase was divided into 3 stages for the CR dosage form (break points at 2 and 4 hours postdose) and only 2 stages for the IR dosage form (break point at 1 hour postdose) (Table 1). This approach resulted in a dramatic improvement in model performance ( 1000 points drop in NONMEM objective function) and had higher impact with the CR dosage form than with the IR dosage form (Table 2). However, the improvement in the model performance with the IR dosage form was statistically significant and cannot be attributed only to increased model flexibility (Table 2). The slow absorption of carvedilol from the IR dosage form during the first hour of administration followed by fast absorption afterward, which resulted in improved model performance with breaking the absorption process into 2 stages (Table 1), may be a result of slow gastric emptying to the duodenum due to administration of the drug under the fed state. Overall, the absorption, as estimated by the rate constants reported in Table 1, was slower at equivalent times and more extended for the CR dosage form when compared with the IR dosage form.

The population estimate of the bioavailability of $S(-)$ carvedilol in the new CR dosage form was $76 \%$ relative to the morning dose of the IR dosage form (Table 1). This value is consistent with the proposed approximate $30 \%$ increase in the CR carvedilol free-base daily dose that was based on calculation of the CR exposure using the traditional noncompartmental analysis. ${ }^{11}$

Carvedilol pharmacokinetics has shown consistent diurnal variability with lower rate of absorption and lower $\mathrm{C}_{\max }$ for the evening administration of IR carvedilol when compared with the morning administration. ${ }^{11}$ Similar diurnal phenomenon was observed with the majority of the IR data analyzed in the present report. Consequently, we assigned different bioavailability factors and absorption rate constants for the morning and evening doses since the difference cannot be regarded only as a consequence of the random variability of the study occasion. The population estimate of bioavailability of the evening IR dose was $80 \%$ relative to the morning IR dose. In addition, the absorption rate constants estimated for the evening dose were significantly lower than for the 
The AAPS Journal 2007; 9 (2) Article 23 (http://www.aapsj.org).

Table 2. Impact of the Modifications Resulting From Single Parameter Deletions From the Final Model on the Fit to the Data (Represented as the Change in the Value of the Objective Function $[\Delta \mathrm{OF}])$

\begin{tabular}{|c|c|c|}
\hline Modification & OF & $\Delta \mathrm{OF}$ \\
\hline Final model & -2490 & - \\
\hline $\begin{array}{l}\text { Break point at } 2 \text { hours for } \mathrm{KA}_{\mathrm{CR}} \\
\text { removed }\end{array}$ & -1983 & +507 \\
\hline $\begin{array}{l}\text { Break point at } 4 \text { hours for } \mathrm{KA}_{\mathrm{CR}} \\
\text { removed }\end{array}$ & -1666 & +824 \\
\hline $\begin{array}{l}\text { Break point at } 1 \text { hour for } \mathrm{KA}_{\text {IRAM }} \\
\text { removed }\end{array}$ & $-2302 *$ & +188 \\
\hline $\begin{array}{l}\text { Break point at } 1 \text { hour for } \mathrm{KA}_{\text {IRPM }} \\
\text { removed }\end{array}$ & -2303 & +187 \\
\hline Single estimated Tlag for CR and IR & -2472 & +18 \\
\hline $\begin{array}{l}\mathrm{F}_{\text {rel }} \text { for CR fixed to } 1 \text { (equals } \mathrm{F}_{\text {rel }} \\
\quad \text { for IR am) }\end{array}$ & -2453 & +37 \\
\hline $\begin{array}{l}\mathrm{F}_{\text {rel }} \text { for IR pm fixed to } 1 \text { (equals } \mathrm{F}_{\text {rel }} \\
\quad \text { for IR am) }\end{array}$ & $-2425^{*}$ & +65 \\
\hline IOV on $\mathrm{KA}_{\mathrm{CR}}$ removed & -1723 & +767 \\
\hline IOV on $\mathrm{F}_{\text {rel } \mathrm{CR}}$ removed & -2460 & +30 \\
\hline ISV on residual error removed & -2371 & +119 \\
\hline
\end{tabular}

KA indicates first-order absorption rate constant; CR, controlled release; IR, immediate release; Tlag, time lag; $F_{\text {rel }}$, relative bioavailability factors; ISV, intersubject variability; IOV, interoccasion variability; not applicable.

*The minimization procedure was not successful (terminated).

morning dose (Table 1). The diurnal variability in the absorption process has been previously reported for several other drugs. ${ }^{19-21}$ This variability may be resulting from the circadian variations in the gastrointestinal functions. ${ }^{22}$

The final model estimates of the intersubject variability in the absorption rate constants of carvedilol were relatively high regardless of the dosage form. Carvedilol is a substrate of the efflux transporters P-glycoprotein (P-gp) and the multidrug resistance associated protein 2 (MRP2). ${ }^{23-25} \mathrm{P}-\mathrm{gp}$ and MRP2 are expressed at the apical surface of the intestinal mucosa and their levels of expression were previously identified among the major variables affecting carvedilol disposition in healthy subjects. ${ }^{26}$ Based on that, it is possible that intersubject variability in the expression of these efflux transporters may have contributed to the high intersubject variability in carvedilol absorption rates. Unfortunately, there are no data regarding the level of expression of these transporters in the studied volunteers to investigate this possibility. Administration of carvedilol under fed conditions, and consequently the expected intersubject variability in gastric emptying and intestinal motility may also have contributed to the high variability in KAs. Interestingly, the model estimate of intersubject variability in the KAs was lower for the CR dosage form when compared with the
Table 3. Evaluation of the Final Model by Leverage Analysis

\begin{tabular}{|c|c|c|}
\hline Parameter & $\begin{array}{c}\text { Mean of } 10 \\
\text { Leverage } \\
\text { Analysis } \\
\text { Runs (\% CV) }\end{array}$ & $\begin{array}{c}\text { Final } \\
\text { Model Point } \\
\text { Estimate }\end{array}$ \\
\hline$\theta_{\mathrm{CL} / \mathrm{F}}(\mathrm{L} / \mathrm{h})$ & $149(2.5)$ & 149 \\
\hline$\theta_{\mathrm{Vc} / \mathrm{F}}(\mathrm{L})$ & $828(4.8)$ & 828 \\
\hline$\theta_{\mathrm{Vp} / \mathrm{F}}(\mathrm{L})$ & $1147(5.9)$ & 1150 \\
\hline$\theta_{\mathrm{Q} / \mathrm{F}}(\mathrm{L} / \mathrm{h})$ & $95(2.9)$ & 94.7 \\
\hline$\theta_{\mathrm{KA}, \mathrm{CR}} 0-2 \mathrm{~h}\left(\mathrm{~h}^{-1}\right)$ & $0.09(11.8)$ & 0.08 \\
\hline$\theta_{\mathrm{KA}, \mathrm{CR}} 2-4 \mathrm{~h}\left(\mathrm{~h}^{-1}\right)$ & $0.29(9.6)$ & 0.27 \\
\hline$\theta_{\mathrm{KA}, \mathrm{CR}}>4 \mathrm{~h}\left(\mathrm{~h}^{-1}\right)$ & $3.83(7.0)$ & 3.50 \\
\hline$\theta_{\mathrm{KA}, \mathrm{IR}, \mathrm{AM}} 0-1 \mathrm{~h}\left(\mathrm{~h}^{-1}\right)$ & $0.92(14.7)$ & 0.92 \\
\hline$\theta_{\mathrm{KA}, \mathrm{IR}, \mathrm{AM}}>1 \mathrm{~h}\left(\mathrm{~h}^{-1}\right)$ & $8.40(37.3)$ & 8.79 \\
\hline$\theta_{\mathrm{KA}, \mathrm{IR}, \mathrm{PM}} 0-1 \mathrm{~h}\left(\mathrm{~h}^{-1}\right)$ & $0.42(14.3)$ & 0.42 \\
\hline$\theta_{\mathrm{KA}, \mathrm{IR}, \mathrm{PM}}>1 \mathrm{~h}\left(\mathrm{~h}^{-1}\right)$ & $3.03(15.1)$ & 3.0 \\
\hline$\theta_{\text {Frel }}, \mathrm{CR}$ & $0.76(5.9)$ & 0.76 \\
\hline$\theta_{\text {Frel, IR, AM }}$ & 1 (Fixed) & 1 (Fixed) \\
\hline$\theta_{\text {Frel, IR, PM }}$ & $0.80(1.9)$ & 0.80 \\
\hline$\theta_{\text {Tlag,CR }}(\mathrm{h})$ & 0.23 (Fixed) & 0.23 (Fixed) \\
\hline$\theta_{\text {Tlag,IR }}(\mathrm{h})$ & $0.20(4.0)$ & 0.20 \\
\hline$\omega \mathrm{Vc} / \mathrm{F} 2$ & $0.14(6.6)$ & 0.14 \\
\hline$\omega \mathrm{KA}, \mathrm{CR} 2$ & $0.98(15.5)$ & 0.90 \\
\hline$\omega \mathrm{KA}, \mathrm{IR}, \mathrm{AM} 2$ & $2.05(12.2)$ & 1.97 \\
\hline$\omega \mathrm{KA}, \mathrm{IR}, \mathrm{PM} 2$ & $3.81(12.7)$ & 3.73 \\
\hline$\omega$ Frel2 & $0.12(5.9)$ & 0.11 \\
\hline$\omega R E S$ ERR2 & $0.05(14.8)$ & 0.05 \\
\hline$\pi \mathrm{KA}, \mathrm{CR} 2$ & $1.47(15.4)$ & 1.29 \\
\hline$\pi$ Frel,CR2 & $0.015(41.3)$ & 0.02 \\
\hline$\sigma^{2}$ & $0.10(3.1)$ & 0.10 \\
\hline
\end{tabular}

CL indicates clearance; Vc, volume of central compartment; Vp, volume of peripheral compartment; $\mathrm{Q}$, intercompartmental clearance; $\mathrm{KA}$, first-order absorption rate constant; $\mathrm{F}_{\text {rel }}$, relative bioavailability factors; Tlag, lag time; CR, controlled release; IR, immediate release.

original IR dosage form. In addition, the evening administration of the IR dosage form showed higher intersubject variability when compared with the morning administration or with the CR dosage form, which may suggest that a oncedaily formulation administered in the morning may be superior to the IR formulation in providing consistent exposure. The clinical significance of this is still unclear.

The final model characterized the pharmacokinetic parameters of carvedilol in both formulations with adequate precision. The point estimate of the oral clearance of $S(-)$-carvedilol from our population model $(149 \mathrm{~L} / \mathrm{h})$ was consistent with the previously reported median value in healthy volunteers $(2439 \mathrm{~mL} / \mathrm{min}$, which is equivalent to $146 \mathrm{~L} / \mathrm{h}$ ) calculated using noncompartmental analysis. ${ }^{9}$ The final population model was evaluated using different methods. The stepwise 
The AAPS Journal 2007; 9 (2) Article 23 (http://www.aapsj.org).

Table 4. Evaluation of the Final Model by Simulation

\begin{tabular}{|c|c|c|c|c|c|c|c|}
\hline \multirow[b]{2}{*}{ Parameter } & & \multicolumn{4}{|c|}{ CR Dosage Form } & \multicolumn{2}{|c|}{ IR Dosage Form } \\
\hline & & $10 \mathrm{mg}$ & $20 \mathrm{mg}$ & $40 \mathrm{mg}$ & $80 \mathrm{mg}$ & $25 \mathrm{mg}$ (am) & $25 \mathrm{mg}(\mathrm{pm})$ \\
\hline \multirow{2}{*}{$\begin{array}{l}\mathrm{AUC}_{0 \text {-tlast }} \\
\quad\left(\mathrm{ng} \mathrm{g}^{*} / \mathrm{mL}\right)^{*}\end{array}$} & Observed median & 12.75 & 31.77 & 71.29 & 145.59 & 59.65 & 83.5 \\
\hline & $90 \%$ prediction interval & $11.66-21.74$ & $27.65-48.67$ & $57.80-98.50$ & $116.73-193.73$ & $48.80-61.39$ & 75.19-93.72 \\
\hline \multirow[t]{2}{*}{$\mathrm{C}_{\max }(\mathrm{ng} / \mathrm{mL})$} & Observed median & 1.81 & 3.76 & 8.13 & 17.87 & 15.65 & 12.6 \\
\hline & $90 \%$ Prediction interval & $1.80-3.22$ & $3.52-6.53$ & $6.99-13.23$ & $14.02-25.75$ & $11.62-15.03$ & $9.54-12.81$ \\
\hline \multirow{2}{*}{$\begin{array}{l}\mathrm{T}_{\max } \\
\text { (h) }\end{array}$} & Observed median & 6 & 5 & 5 & 5 & 1.5 & 14 \\
\hline & $90 \%$ Prediction interval & $4-6$ & $4.5-6$ & $4.5-6$ & $4.5-6$ & $1.5-2$ & $13.5-14$ \\
\hline \multirow[t]{2}{*}{$\mathrm{C}_{\min }(\mathrm{ng} / \mathrm{mL})^{\dagger}$} & Observed median & 0.29 & 0.41 & 0.93 & 1.81 & 1.49 & 2.2 \\
\hline & $90 \%$ Prediction interval & $0.23-0.40$ & $0.31-0.59$ & $0.57-1.11$ & $1.11-2.12$ & $1.32-1.75$ & $1.78-2.38$ \\
\hline
\end{tabular}

CR indicates controlled release; IR, immediate release.

*The reported AUC was calculated from 0-12, 12-48, and 0-48 hours for the $\mathrm{IR}_{\mathrm{AM}}, \mathrm{IR}_{\mathrm{PM}}$, and CR doses respectively.

${ }^{\dagger} \mathrm{C}_{\min }$ was calculated as $\mathrm{C}_{12}$ hour for the $\mathrm{IR}_{\mathrm{AM}}$ dose and as $\mathrm{C}_{24}$ hour for the $\mathrm{IR}_{\mathrm{PM}}$ dose and the $\mathrm{CR}$ doses.

The $90 \%$ prediction intervals were constructed by calculating the 5 th and the 95 th percentiles of the medians for the 500 simulated replicates.

deletion of parameters from the model indicated that all the parameters included in the final model were required to describe the observed trends in the data and that the model was not over parameterized. The results of leverage analysis indicated the robustness of the final model to outliers as was evident by the agreement between the mean of the parameter estimates from the 10 leverage analysis runs (in each of which $30 \%$ of the subjects were randomly deleted) and the parameter point estimates for the whole data set. In addition, the results of the internal evaluation of the predictive ability of the model using Monte Carlo simulations indicated that the model replicated the salient features of the data from which it was built.

\section{CONCLUSION}

In summary, we developed a single population model that describes the pharmacokinetics of the IR and CR dosage forms of carvedilol in healthy volunteers. The model was used to compare the absorption processes of both dosage forms as well as to estimate the intersubject variability and interoccasion variability in the pharmacokinetic parameters. The model showed satisfactory performance in internal evaluation and will be a useful tool for future simulation studies.

\section{REFERENCES}

1. Ruffolo RR, Jr, Boyle DA, Venuti RP, Lukas MA. Preclinical and clinical pharmacology of carvedilol. J Hum Hypertens. 1993;7:S2-15.

2. Ruffolo RR, Jr, Gellai M, Hieble JP, Willette RN, Nichols AJ.

The pharmacology of carvedilol. Eur J Clin Pharmacol. 1990;38:S82-S88.

3. van Zwieten PA. Pharmacodynamic profile of carvedilol. Cardiology. 1993;82:19-23.

4. Colucci WS, Packer M, Bristow MR, et al. Carvedilol inhibits clinical progression in patients with mild symptoms of heart failure.
US Carvedilol Heart Failure Study Group. Circulation. 1996;94:2800-2806.

5. Packer M, Bristow MR, Cohn JN, et al. The effect of carvedilol on morbidity and mortality in patients with chronic heart failure. US Carvedilol Heart Failure Study Group. $N$ Engl J Med. 1996;334:1349-1355.

6. Dargie HJ. Effect of carvedilol on outcome after myocardial infarction in patients with left-ventricular dysfunction: the CAPRICORN randomised trial. Lancet. 2001;357:1385-1390.

7. Bartsch W, Sponer G, Strein K, et al. Pharmacological characteristics of the stereoisomers of carvedilol. Eur J Clin Pharmacol. 1990;38:S104-S107.

8. Ruffolo RR, Boyle DA, Venuti RP, Lukas M. Carvedilol (Kedrex $\left.{ }^{\circledR}\right)$ : a novel multiple action cardiovascular agent. Drugs Today. 1991;27:465-492.

9. Neugebauer G, Akpan W, Kaufmann B, Reiff K. Stereoselective disposition of carvedilol in man after intravenous and oral administration of the racemic compound. Eur J Clin Pharmacol. 1990;38:S108-S111.

10. Zhou HH, Wood AJ. Stereoselective disposition of carvedilol is determined by CYP2D6. Clin Pharmacol Ther. 1995;57:518-524.

11. Tenero DM, Henderson LS, Baidoo CA, et al. Pharmacokinetic properties of a new controlled-release formulation of carvedilol. Am J Cardiol. 2006;98:5-16.

12. Packer M, Lukas MA, Tenero DM, Baidoo CA, Greenberg BH. Pharmacokinetic profile of controlled-release carvedilol in patients with left ventricular dysfunction associated with chronic heart failure or after myocardial infarction. Am J Cardiol. 2006;98:39-45.

13. Weber MA, Sica DA, Tarka EA, Iyengar M, Fleck R, Bakris GL. Controlled-release carvedilol in the treatment of essential hypertension. Am J Cardiol. 2006;98:32-38.

14. Yang E, Wang S, Kratz J, Cyronak MJ. Stereoselective analysis of carvedilol in human plasma using HPLC/MS/MS after chiral derivatization. J Pharm Biomed Anal. 2004;36:609-615.

15. Karlsson MO, Sheiner LB. The importance of modeling interoccasion variability in population pharmacokinetic analyses. J Pharmacokinet Biopharm. 1993;21:735-750.

16. Karlsson MO, Jonsson EN, Wiltse CG, Wade JR. Assumption testing in population pharmacokinetic models: illustrated with an 
The AAPS Journal 2007; 9 (2) Article 23 (http://www.aapsj.org).

analysis of moxonidine data from congestive heart failure patients. J Pharmacokinet Biopharm. 1998;26:207-246.

17. Sheiner LB, Ludden TM. Population pharmacokinetics/dynamics. Annu Rev Pharmacol Toxicol. 1992;32:185-209.

18. Beal SL, Sheiner LB. NONMEM Users Guide, Part V. San Francisco, CA: Division of Clinical Pharmacology, University of California, San Francisco; 1979-1992.

19. Park SI, Jr, Felipe CR, Jr, Pinheiro-Machado PG, Jr, Garcia R, Jr, Tedesco-Silva H, Jr, Medina-Pestana JO. Circadian and time-dependent variability in tacrolimus pharmacokinetics. Fundam Clin Pharmacol. 2007;21:191-197.

20. Aronson JK, Chappell MJ, Godfrey KR, Yew MK. Modelling circadian variation in the pharmacokinetics of non-steroidal antiinflammatory drugs. Eur J Clin Pharmacol. 1993;45:357-361.

21. Milanian I, Ghods AJ, Mahmoudian M, et al. Study of circadian variation of cyclosporine pharmacokinetics. Transplant Proc. 1997;29:2930-2931.
22. Vener KJ, Moore JG. Chronobiologic properties of the alimentary canal affecting xenobiotic absorption. Ann Rev Chronopharmacol. 1987;4:257-281.

23. De Mey C, Brendel E, Enterling D. Carvedilol increases the systemic bioavailability of oral digoxin. Br J Clin Pharmacol. 1990;29:486-490.

24. Jonsson O, Behnam-Motlagh P, Persson M, Henriksson R, Grankvist K. Increase in doxorubicin cytotoxicity by carvedilol inhibition of P-glycoprotein activity. Biochem Pharmacol. 1999;58:1801-1806.

25. Kaijser M, Johnsson C, Zezina L, Backman U, Dimeny E, Fellstrom B. Elevation of cyclosporin A blood levels during carvedilol treatment in renal transplant patients. Clin Transplant. 1997;11:577-581.

26. Giessmann T, Modess C, Hecker U, et al. CYP2D6 genotype and induction of intestinal drug transporters by rifampin predict presystemic clearance of carvedilol in healthy subjects. Clin Pharmacol Ther. 2004;75:213-222. 